\title{
The development of rotational molding of waste bamboo powder with LLDPE plastic powder
}

\author{
Tapragon Ouprara ${ }^{1 *}$ and Kiattisak Sangpradit ${ }^{2}$ \\ Rajamangala University of Technology Thanyaburi Department of Agricultural Engineering, 39 \\ Moo1 Klong 6, Thanyaburi, Pathum Thani, 12110,Thailand
}

\begin{abstract}
The objective of this study was to assess the effect of bamboo powder addition and the treatment of its surface on the properties and structure of rotationally molded (rotomolded) polypropylene. In this study we can transform plastic and bamboo powder into a product by putting the raw materials into hollow mold and then cover the mold. The mold is rotated along two axes by burning with high temperature to make liquid plastic flows evenly along the inner surface of the mold with gravity. The results of this research had shown that, by using LLDPE plastic $4 \mathrm{~kg}$, bamboo waste powder $1 \mathrm{~kg}$ size of $0.15: 0.30: 0.85 \mathrm{~mm}$ tested with humidity $10: 15: 20 \%$ mixed and then molded, it was found that the moisture of waste bamboo powder had a direct effect on molding. If the moisture in the bamboo powder is more than $20 \%$, the work will not be molded. It shown look like a lump. The moisture content less than $15 \%$ can be molded as a product. In terms of the size used in this test $0.15: 0.30: 0.85$ can be molded well in the range of moisture, not more than $15 \%$.
\end{abstract}

\section{Introduction}

Rotational molding is a widely use technological process for the production of thin walled products, usually characterized by large dimensions. The main advantages in comparison to alternative processes are the technological machine as well as high technology flexibility. Simultaneously one of the biggest disadvantage accompanying to production of rotomolded casting is the necessity of using material with the specified with geometrical characteristic and processing properties, especially rheological properties [1]. Rotational moulding is a closed-mould plastics manufacturing process for producing large hollow objects. Each production cycle comprises of four stages: material charging, heating, cooling and demoulding, with the mould rotating bi-axially during both the heating and cooling stages. The rapid development of this process as a mainstream plastics manufacturing method is attributed to its many advantages, such as the absence of residual stresses, the low manufacturing and material costs, and the capability of manufacturing large and high quality products. However, some limitations also exist such as the high mould cost, the long processing times, the limited mouldable materials and the mould shape complexity. More than $85 \%$ of the raw materials used in the rotomoulding industry worldwide are the linear polyethylenes (both low and medium densities) due to their thermal durability in the

* Corresponding author: tapragon@gmail.com 
prolonged heating environment [2]. However, these materials possess modest mechanical properties which prevent them from being used in more advanced applications. Therefore, the introduction of reinforcements in the rotomoulding process in order to obtain stronger/stiffer rotomoulded products has attracted increasing attention [3].

The processing of this research is to develop waste bamboo processing in order to be friendly to the environment. It helps to protect the environment and reduce air pollution that will cause PM 2.5 by burning the agricultural crops. This is a global problem at this time as well. By using bamboo powder blended with LLDPE polyethylene and then molded by the roto mold process to produce products in various forms. The community together with entrepreneurs will help to develop and solve the problem of waste caused by agricultural products. In another way from bamboo processing, from visiting a bamboo processing factory and meeting to find a solution for the development of waste from bamboo processing of farmers with the objective to develop composite materials from waste bamboo o be able to further transform and create waste bamboo products by process rotomolded plastic rotational molding such as making a compact shape, planting trees to further expand agricultural value products.

\section{Materials and methods}

Forming waste bamboo powder mixed with LLDPE plastic by the rotomold vertical rotation process it is a simple method, not complicated with working principles. The process consists of the following main steps: adding $1.00 \mathrm{~kg}$ of waste bamboo powder mixed with plastic powder (loading) LLDPE $4 \mathrm{~kg}$ mixed and then put into the mold, after close the lid, sandwich the mold. Forming or melting (molding or curing). Move the mold into the hot room to rotate two cores along with heating to make liquid plastic and flows evenly along the inner surface of the mold with gravity (not centrifugal) and then cooling (cooling) using a fan to cool the mold. But the mold must still be rotated in order to reduce the shrinkage of the work piece during cooling and unloading. After that, the work hardens and maintains its shape can open the mold to take out the work piece repeat this test repeatedly to get results. A-B-C-D-E-FG-H-I with the test procedure as follows:

\subsection{Sources of waste bamboo powder}

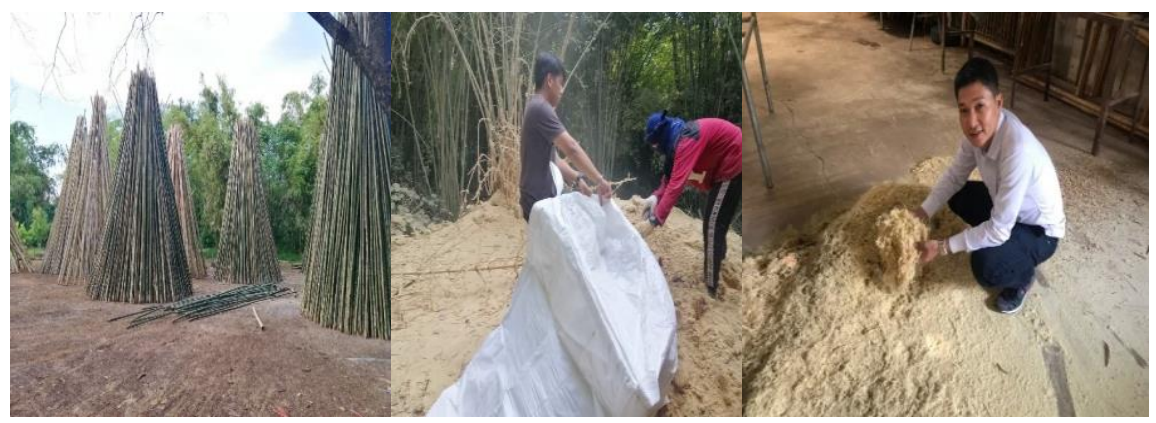

Fig. 1. Picture of the remaining bamboo powder area. 


\subsection{Sorting waste bamboo powder}

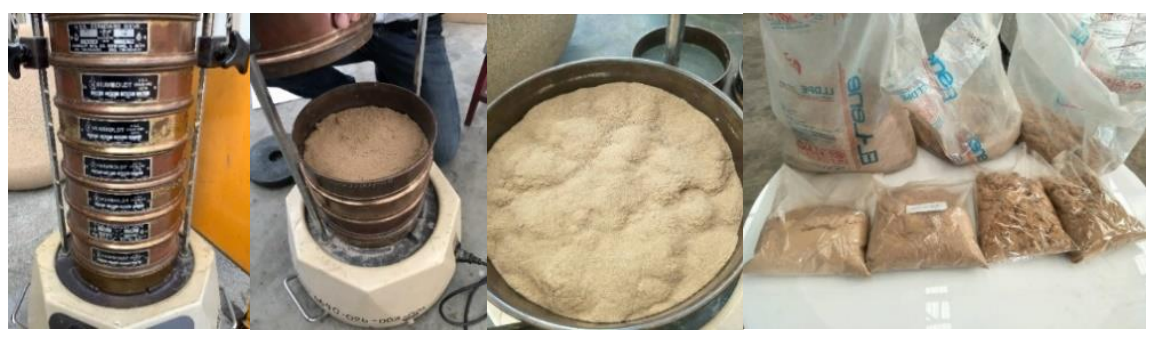

Fig. 2.Picture of size-separating waste bamboo.

\subsection{Mixing bamboo powder with recycled plastic and bio plastic}

The materials were weighed then mixed at the ratio of LLDPE; plastic $4.00 \mathrm{~kg}$, bamboo waste $1.00 \mathrm{~kg}$, then mixed together.

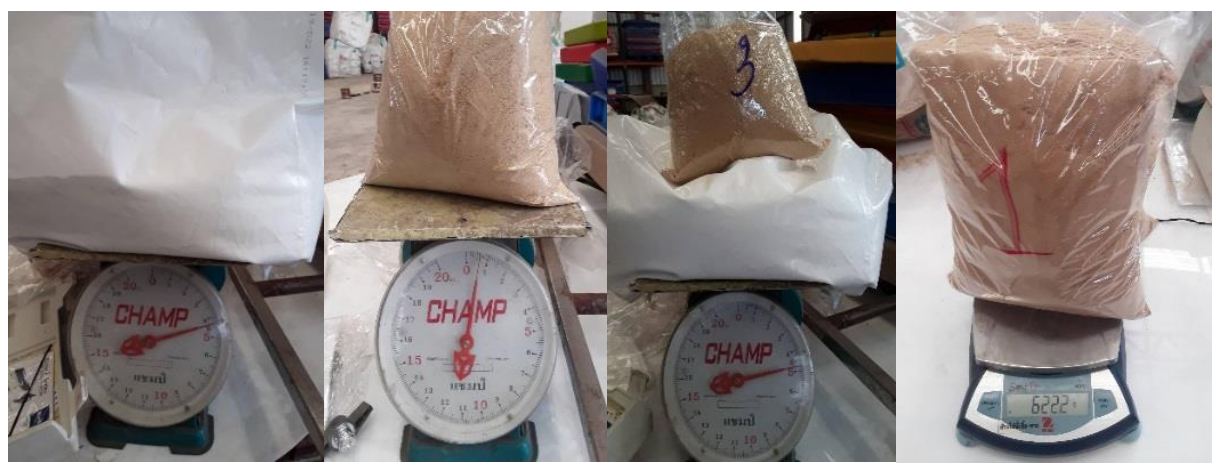

Fig. 3.Picture of weighing material.

\subsection{Measuring humidity of bamboo powder}

Moisture of bamboo powder was measured with Long Rice Moisture Tester Riceter f514

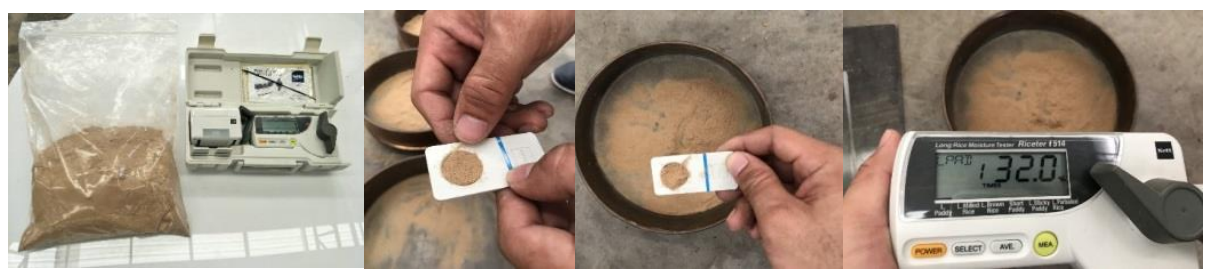

Fig. 4.Picture of measuring satisfaction. 


\subsection{Forming process by using the roto-mold}

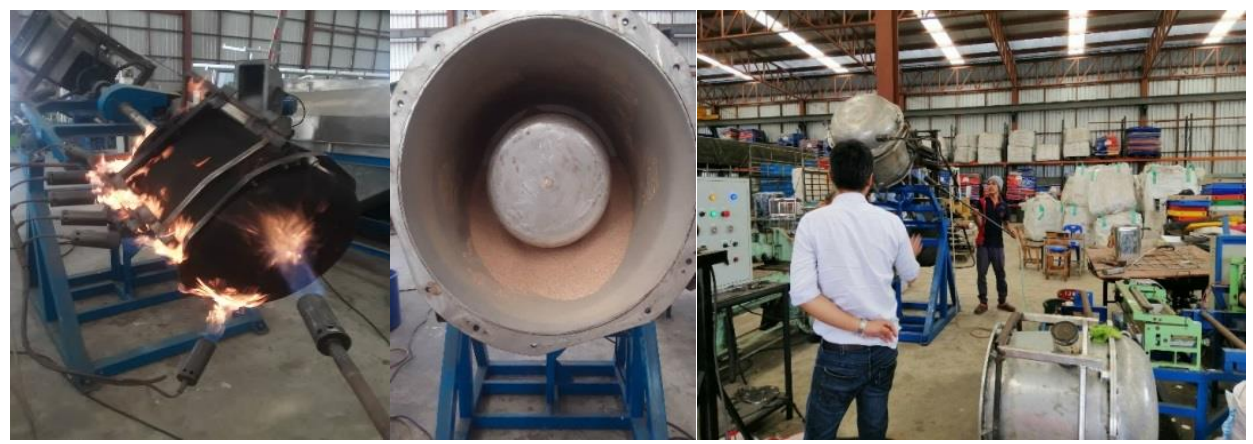

Fig. 5.Forming test of the product.

\section{Results and discussion}

Table. 1. Variation of experimental results.

\begin{tabular}{|c|c|c|c|}
\hline Set & Moisture (\%) & Size of bamboo powder $(\mathrm{mm})$ & Results \\
\hline A & 10 & 0.15 & $\mathrm{C}$ \\
\hline B & 10 & 0.30 & $\mathrm{C}$ \\
\hline C & 10 & 0.85 & $\mathrm{C}$ \\
\hline D & 15 & 0.15 & $\mathrm{C}$ \\
\hline E & 15 & 0.30 & $\mathrm{C}$ \\
\hline F & 15 & 0.85 & $\mathrm{C}$ \\
\hline G & 20 & 0.15 & N/A \\
\hline H & 20 & 0.30 & N/A \\
\hline I & 20 & 0.85 & N/A \\
\hline
\end{tabular}

From the table above shown that, by testing of $20 \mathrm{rev} / \mathrm{mn}$ at the horizontal axis rotation of workpiece using $1 \mathrm{hp}$ motor for the main power, and speed around the vertical axis $60 \mathrm{rev} /$ $\mathrm{mn}$ using $2 \mathrm{hp}$ motor for the main power with the moisture and size of bamboo powder is found that; plastic molding with bamboo powder by dividing into 3 steps: A, B, C (Fix), 10\% of moisture, 0.15,0.30,0.85 of bamboo powder size can be molded (C). D, E, F (Fix), 15\% of moisture with the $0.15,0.30,0.85$ of bamboo powder size can be molded (C). And G, H, I, $20 \%$ of moisture with the same size of powder cannot be molded (N/A). It shows that the moisture of the bamboo powder affects the forming of the workpiece.

$\mathrm{C}=$ Complete

N/A $=$ Not applicable 


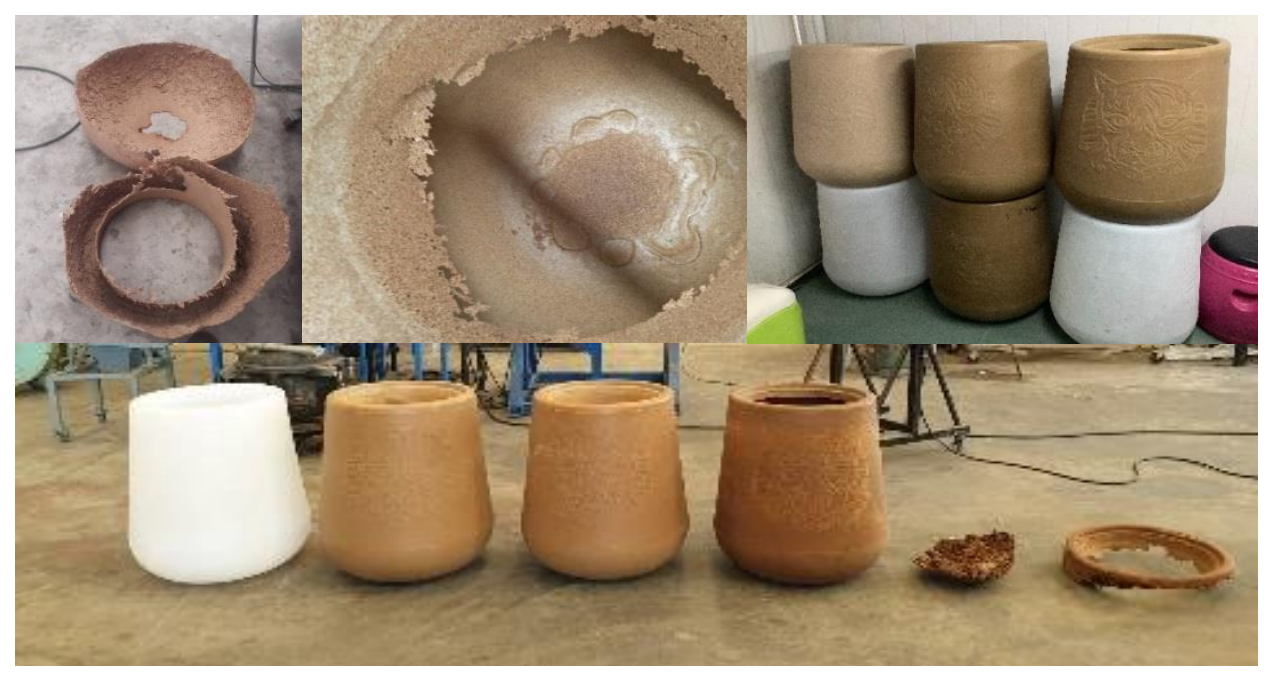

Fig. 5. Samples of product by using a rotary molding process from LLDPE mixed with bamboo powder.

\section{Conclusions}

According to the results found that bamboo powder can be molded into the roto-molding process. By mixing with LLDPE plastic, it helps reduce waste of bamboo waste which can be processed into products and benefit the value of processing. The main factor in forming is moisture. The moldable moisture content is not more than $15 \%$. If moisture content is more than $15 \%$, it cannot be molded. The size of bamboo powder also affects by the size of bamboo powder tested 0.15: 0.30: $0.85 \mathrm{~mm}$ within the range of forming well but if the powder size is bigger than this, the bamboo and plastic powder parts will be clearly separated where the large powder is on the inside and the small powder is on the outside of the tank that will allowed us to see the difference.

The author would like to thanks The Center of Robotics and Precision Farming for cooperation for this research.

\section{References}

1. R.J. Crawford, J.L. Throne, Rotational Molding Technology, Plastics Design Library (William Andrew Publishing, Norwich, New York ,2001)

2. R.J. Crawford, Rotational moulding of plastics (Research Studies Press, Somerset, UK, 1996)

3. W. Yan, R.J.T. LinM and D. Bhattacharyya, Composites Science and Technology 66, 2080 (2006)

4. S. Mandal, S. Alam, IK. Verma,SN. Maiti, J Reinf Plast Compos 29, 43-51 (2010) 